\title{
THE CORRELATION BETWEEN THE EIGENVALUES \\ OF GLOBAL FEM MATRIX AND THE SIZE OF TIME STEP IN THE NUMERICAL SOLUTIONS OF SOLIDIFICATION EQUATION
}

\author{
Maria Zych \\ Institute of Computer and Information Science, Czestochowa University of Technology \\ Częstochowa, Poland \\ maria.zych@icis.pcz.pl
}

\begin{abstract}
In the paper the influence of the eigenvalues of the matrix on the stability of the numerical simulations of binary alloy solidification process was analyzed. One step $\Theta$ scheme time integration for the modeling was used. The occurrence of the equation clotting full, diagonal and lumped capacitance matrix was considered. In the explicit schema (conditionally stable) the major influence on the proper selection of the size of the critical time step was proved. The physically incorrect results are given when the maximum allowable time step is exceeded.
\end{abstract}

Keywords: eigenvalues of the matrix, time step, numerical stability, solidification

\section{Introduction}

Eigenvalues of the matrix used in numerical simulations has been well known, for instance, in the solidification process. The paper focuses on the numerical model of the matrix including eigenvalues.

The solidification phenomenon is described by the equation of heat conduction:

$$
c \rho \frac{\partial T}{\partial T}-\nabla \cdot(\lambda \nabla T)=\rho_{s} L \frac{\partial f_{s}}{\partial t},
$$

where $T$ - the temperature, $t$ - the time, $\lambda$ - the thermal conductivity, $\rho_{s}$ - the density of the solid phase, $L$ - the latent heat of solidification, $f_{s}$ - solid phase content defined as the quotient of the volume of solidified and total volume of the grain after solidification. In the mold: $f_{s}=1=$ const, and in the liquid: $f_{s}=0=$ const.

When the right side of equation (1) is equal to null, the pattern is reduced to the ordinary heat conduction equation without source. Equation (1) with the initial and boundary conditions is the basis of the temperature description of solidification [1].

In order to describe the enthalpy spin of the solidification, the concept of enthalpy has been introduced [2]: 


$$
H(T)=\int_{T_{r e f}}^{T} c \rho(T) d T+\rho_{s} L\left(1-f_{s}(T)\right),
$$

where $T_{\text {ref }}$ is the reference temperature and $c \rho$ is the heat capacity, one can pass to the so-called enthalpy description of the solidification process. A few types of enthalpy formulations of solidification exist. One of them is so-called apparent heat capacity formulation:

$$
\nabla \cdot(\lambda \nabla T)=c^{*} \frac{\partial T}{\partial t},
$$

containing the effective heat capacity $\left(c^{*}\right)$, which is obtained by differentiating equations (2) with respect to temperature [2]:

$$
\frac{d H}{d T}=c \rho-\rho_{s} L \frac{d f_{s}}{d T}=c^{*}(T) .
$$

Using the Finite Element Method, the equation (3) is converted to an ordinary differential equation with a derivative of temperature with respect to time:

$$
\mathbf{M}(T) \dot{\mathbf{T}}+\mathbf{K}(T) \mathbf{T}=\mathbf{b}(T),
$$

where $\mathbf{M}$ is capacity matrix, $\mathbf{K}$ - the conductivity matrix, $\mathbf{T}$ - the temperature vector. Vector $\mathbf{b}(T)$ is computed on the basis of the boundary conditions.

The $\theta$ Euler integration over time scheme is chosen for the numerical integration. When $\theta=1$ then the scheme is implicit and unconditionally stable. If $\theta=0$ then the scheme is explicit and conditionally stable. Conditional stability is determined by the size of the time step. In turn, the time step size is limited by the eigenvalues of the matrices $\mathbf{M}$ and $\mathbf{K}$.

For equation (5) in the matrix form, the correlation between the eigenvalues of the matrix and the size of the time step for three different matrix capacities (full, diagonal and lumped) [3] will be considered.

\section{Reducing the size of the time step}

The numerical method is called stable if the error at any stage of solution moves further from the declining value. Numerical stability is reached when the condition is fulfilled:

$$
|g| \leq 1
$$

where $g$ is a gain factor associated with the integration scheme over time. Condition (6) is limited to the finite issues.

To determine the stability criterion of the numerical method, the transformative operations on the matrix equation of the freezing point to the general issue of its 
eigenvalues is used. Depending on the form of matrix $\mathbf{M}$ (full, diagonal, lumped), the method of determining the inverse matrix is a more or less complicated process. In the case of a diagonal matrix, the inversion and calculation of the eigenvalues is not a problem. Eigenvalues can be listed from the smallest to the largest and designate the time step on its basis.

If the matrix $\mathbf{M}$ is a matrix of full inversion, the above described process is not trivial and requires the use of various converting operations.

The correct mathematical methods in this case are the Cholesky decomposition and the Rayleigh quotient. Using these equations provides the scalar equation [4].

Equation (5) obtained as a result of spatial discretization, after using one-step integration scheme is transformed to following form:

$$
(\mathbf{M}+\theta \Delta t \mathbf{K}) \mathbf{T}^{n+1}=(\mathbf{M}-(1-\theta) \Delta t \mathbf{K}) \mathbf{T}^{n} .
$$

In the case when we put $\theta=0$ (explicat shame), the size restrictions of the timestep in the analysis of the numerical stability result from eigenvalues of the Rayleigh quotient [4]. For numerical stability, of explicit schema, the homogeneous part of equation (5) is only considered:

$$
\mathbf{M} \dot{\mathbf{T}}+\mathbf{K T}=0,
$$

which after the transformations related to the eigenvalues provides:

$$
\lambda \mathbf{M v}=\mathbf{K v} .
$$

Because of the semi-discretization, the equation (9) is achieved for $\lambda=\lambda_{i}$ and $\boldsymbol{v}=\boldsymbol{v}_{\boldsymbol{i}}$, where $\boldsymbol{i}$ is the $\boldsymbol{i}$-th element of the grid.

Because of the physical phenomenon, the $\mathbf{M}$ matrix is positively defined and the Cholesky decomposition can be done; so that: $\mathbf{M}=\mathbf{L} \mathbf{L}^{T}$ where $\mathbf{L}$ is a lower triangular and a non-singular matrix.

Using such a distribution in equation (7) and multiplying both sides of the equation by $\mathbf{L}^{-1}$ provides:

$$
\lambda_{i} \mathbf{L}^{T} \mathbf{v}_{i}=\mathbf{L}^{-1} \mathbf{K}\left(\mathbf{L}^{-1}\right)^{T} \mathbf{L}^{T} \mathbf{v}_{i}
$$

where $\mathbf{L}^{T} \boldsymbol{v}_{i}$ is the eigenvector and $\lambda$ is the eigenvalue of a symmetric matrix $\mathbf{M}=\mathbf{L}^{-1} \mathbf{K}\left(\mathbf{L}^{-1}\right)^{T}$. The $\mathbf{M}$ matrix is a set of linearly independent eigenvectors $\boldsymbol{v}_{i}$.

If the matrix $\mathbf{V}$ is composed of $\boldsymbol{v}_{i}$ being the columns of the matrix and $\mathbf{L}^{T} \mathbf{V}$ is orthogonal, the following formula can be formed:

$$
\mathbf{V}^{T} \mathbf{L} \mathbf{L}^{T} \mathbf{V}=\mathbf{V}^{T} \mathbf{M V}=\mathbf{I} .
$$

Equation (9) and Cholesky decomposition process provides:

$$
\lambda_{i}=\lambda_{i} \mathbf{v}_{i}^{T} \mathbf{M} \mathbf{v}_{i}=\mathbf{v}_{i}^{T} \mathbf{K} \mathbf{v}_{i} .
$$


Substituting $\mathbf{T}=\mathbf{V} \mathbf{x}$ to the equation 7 and left-multiplying it by $\mathbf{V}^{T}$, the following formulas are obtained:

$$
\mathbf{V}^{T} \mathbf{M V} \dot{\mathbf{x}}+\mathbf{V}^{T} \mathbf{K V x}=\mathbf{0},
$$

and

$$
\mathbf{I} \mathbf{x}+\Lambda \mathbf{x}=\mathbf{0}
$$

where $\boldsymbol{\Lambda}=\operatorname{diag}\left(\lambda_{i}\right)$. This distribution is known as the decomposition modeling, which makes it possible to save the matrix equations in scalar form:

$$
\dot{x}_{i}+\lambda_{i} x_{i}=0
$$

For the issues described by the equation (9), the eigenvalues and corresponding eigenvectors can be determined. In contrast, the eigenvalues for the equation (12) can be derived from the Rayleigh quotient:

$$
\lambda=\frac{\mathbf{v}^{T} \mathbf{K} \mathbf{v}}{\mathbf{v}^{T} \mathbf{M v}}
$$

After appropriate transformations in which the assembling of the matrix method is included, the constraints of eigenvalues resulting from the Rayleigh quotient is obtained. It has the following form:

$$
\lambda_{\min }^{(e)} \leq \lambda \leq \lambda_{\max }^{(e)} .
$$

From the inequality (17) the largest eigenvalue of the matrix is selected. On the basis of that matrix, the size of the critical time-step used later in numerical simulations of solidification is determined.

\section{Results}

In the calculation the capacity matrix (as a full, diagonal and lumped) was considered. At the end of calculations the oscillations occur for the full matrix of numerical simulation of the solidification process. The whole simulation is run properly and gives results consistent with the physics of the phenomenon $[4,5]$ for the lumped matrix.

The matrix equation of solidification was the base calculation. After appropriate transformations, as well as taking into account the sufficient condition for the stability of the numerical integration scheme [3], the following equation is obtained:

$$
\dot{\mathbf{T}}+\mathbf{B T}=\mathbf{0} .
$$


Knowing that the eigenvalues limit the size of a time-step, the eigenvalues of the $\mathbf{B}$ matrix are determined providing:

$$
\mathbf{B}=\mathbf{M}^{-1} \mathbf{K}
$$

The following three forms of capacity matrices were used in these calculations:

a) full:

$$
\left(\begin{array}{lll}
2 & 1 & 1 \\
1 & 2 & 1 \\
1 & 1 & 2
\end{array}\right)
$$

eigenvalues of $1 ; 4 ; 1$

b) diagonal:

$$
\left(\begin{array}{lll}
1 & 0 & 0 \\
0 & 4 & 0 \\
0 & 0 & 1
\end{array}\right)
$$

eigenvalues of $1 ; 4 ; 1$

c) lumped:

$$
\left(\begin{array}{lll}
1 & 0 & 0 \\
0 & 1 & 0 \\
0 & 0 & 1
\end{array}\right)
$$

eigenvalues of $1 ; 1 ; 1$

The second round of calculations was related to determining the value of their own capacity for the invertible matrix in the order of the calculations in the first round:
a) $1 ; 0.25 ; 1$
b) $1 ; 0.25 ; 1$
c) $1 ; 1 ; 1$

The obtained results prove that the ratio of the eigenvalues of the full matrix to the eigenvalues of the lumped matrix is 4:1 [6]. Therefore, the time-step determined on the basis of the eigenvalues' values is also subject to the same limitation. This situation is reflected in the normal course of calculation in the numerical simulations of the solidification. For the simulations carried out with the time-step set based on eigenvalues of the lumped matrix, the calculations are always stable. However, if the numerical solidification of the simulation process are carried out to the full capacity matrix, the time-step size should be limited by the maximum eigenvalue of the matrix for the final stage not to cause oscillation. 


\section{References}

[1] Sczygiol N., Modelowanie numeryczne zjawisk termomechanicznych w krzepnącym odlewie i formie odlewniczej, Wydawnictwo Politechniki Częstochowskiej, Częstochowa 2000.

[2] Sczygiol N., Szwarc G., Application of enthalpy formulations from numerical simulation of castings solidification, Computer Assisted Mechanics and Engineering Sciences 2011, 8, 99-120.

[3] Shen R.Wu, Lumped mass matrix in explicit finite element method for transient dynamics of elasticity, Comput. Methods Appl. Mech. Engrg. 2006, 195, 5983-5994.

[4] Gawrońska E., Sczygiol N., Stability of the mixed time partitioning methods in relation to the size of time step, Special Issue: 82nd Annual Meeting of the International Association of Applied Mathematics and Mechanics (Gesellschaft für Angewandte Mathematik und Mechanik), GAMM 2011, 18-21 April 2011, Graz, Austria, 467-468.

[5] Gawrońska E., Sczygiol N., Application of mixed time partitioning methods to raise the efficiency of solidification modeling, 12th International Symposium on Symbolic and Numeric Algorithms for Scientific Computing, SYNASC 2010, 23-26 September 2010, Timisoara, Romania, 99-103.

[6] Wood W.L., Partical Time-stepping Schemes, Clarendon Press, Oxford 1990. 